\title{
Avaliação da implantação de escala de dor em uma unidade de terapia intensiva pediátrica
}

\author{
Evaluation of the implantation of pain scale in a pediatric intensive therapy unit \\ Evaluación de la implantación de escala de dolor en una unidad de terapia intensiva \\ pediátrica \\ Beatriz Molina Carvalho ${ }^{1 *}$, Cássia Grigini Godoi², Raquel Matioli Vieira ${ }^{1}$, Larissa Grispan e Silva \\ Guimarães ${ }^{3}$, Mauren Teresa Grubisich Mendes Tacla ${ }^{4}$, Rosângela Aparecida Pimenta Ferrari ${ }^{4}$, \\ Flávia Lopes Gabani ${ }^{4}$.
}

\section{RESUMO}

Objetivo: Avaliar a utilização da Escala Face, Legs, Activity, Cry, Consolability (FLACC) pela equipe de enfermagem como instrumento para manejo da dor de crianças internadas em uma Unidade de Terapia Intensiva Pediátrica (UTIP). Métodos: Estudo retrospectivo e descritivo, abordagem quantitativa. Dados obtidos pela análise de 62 prontuários das crianças com idade entre zero a sete anos incompletos, internadas na UTIP em dois períodos: três meses imediatos à implantação da FLACC e um ano após. Os resultados foram analisados pelo software IBM SPSS Statistics 20. Pesquisa foi aprovada pelo Comitê de Ética em Pesquisa Envolvendo Seres Humanos da Universidade Estadual de Londrina, com parecer ํㅜ 1.627.996, de 07 de julho de 2016, CAAE ํo 56739316.1.0000.5231. Resultados: Na implantação em 31,82\% dos prontuários foi encontrado relato do processo doloroso. Um ano após, em $8,57 \%$ os relatos foram encontrados. Para tratamento da dor foram encontrados registros do uso de analgésicos de diferentes classes. Em nenhum prontuário foi encontrado relato do uso de medidas não farmacológicas. Conclusão: Evidencia-se a necessidade de orientação e capacitação da equipe de modo contínuo e periódico para que os profissionais estejam aptos para reconhecer o processo doloroso e conduzi-lo de modo eficaz, oferecendo assistência qualificada e humanizada à criança.

Palavras-chave: Saúde da Criança, Cuidados Críticos, Enfermagem Pediátrica.

\begin{abstract}
Objective: To evaluate the use of Face, Legs, Activity, Cry, Consolability (FLACC) scales by the nursing staff as an instrument for pain management of hospitalized children in a Pediatric Intensive Care Unit (PICU). Methods: Descriptive retrospective study with a quantitative approach. Data base was collected through the analysis of 62 patient records between the ages zero to seven years old uncompleted, admitted in the PICU in two periods: three months and a year after the implantation of the FLACC scale. The results were analyzed by the IBM SPSS Statistic 20 software. Research approved by the Ethics in Research Involving Human Beings Committee of the State University of Londrina, o 1.627.996, July $7^{\text {th }} 2016$, CAAE $n^{\circ}$ 56739316.1.0000.5231. Results: In the records $31,82 \%$ pain related reports were found in the implantation. A year later $8,57 \%$ pain reports were found. None of the records showed non pharmacological methods. Conclusion: The necessity of staff orientation and training continuously and regularly is evidenced. Therefore, the professionals will be able to identify and evaluate the pain process efficiently, offering a qualified and humanized assistance.
\end{abstract}

Keywords: Child Health, Critical Care, Pediatric Nursing.

${ }^{1}$ Enfermeira Residente em Saúde da Criança pela Universidade Estadual de Londrina (UEL). LondrinaParaná. *E-mail: beatriz.molinacarv@hotmail.com

²Enfermeira Especialista em Enfermagem em Saúde da Criança - Modalidade Residência pela UEL.

3Mestre em Enfermagem. Servidora da Secretaria de Saúde do Estado do Paraná.

${ }^{4}$ Professor Associado do Departamento de Enfermagem da UEL - Área de Saúde da Criança.

SUBMETIDO EM: 2/2019

ACEITO EM: 3/2019

PUBLICADO EM: 4/2019 


\section{RESUMEN}

Objetivo: Evaluarel uso de la Escala Face, Legs, Activity, Cry, Consolability (FLACC) por el equipo de enfermería como instrumento para manejo deldolor de niños internados en Unidad de Terapia Intensiva Pediátrica (UTIP). Métodos: Estudio retrospectivo, descriptivo, enfoque cuantitativo. Datos obtenidos poranálisis de 62 prontuarios de niños entre cero a siete años, internados em la UTIP en dos períodos: tres meses inmediatos a la implantación de la FLACC y um año después. Los resultados fueron analizados por software IBM SPSS Statistics 20. Investigación aprobada por el Comité de Ética em Investigación Involucrando Seres Humanos de la Universidade Estadual de Londrina, dictamen № 1.627.996, de 07/07/2016, CAAE no 56739316.1.0000.5231. Resultados: Em la implantaciónen 31,82\% de los prontuários fue encontrado relato del proceso doloroso. Um año después, enel 8,57\% se encontraron los relatos. Para el tratamiento del dolor habia registros del uso de analgésicos de diferentes clases. Em ningún prontuario se encontró relato del uso de medidas no farmacológicas. Conclusión: Se evidencia necesidad de orientación y capacitación del equipo de modo continuo y periódico para que los profesionales estén aptos para reconocer el proceso doloroso y conducirlo de manera eficaz, ofereciendo asistencia calificada y humanizada al niño.

Palavras-clave: Salud del Niño, Cuidados Críticos, Enfermería Pediátrica.

\section{INTRODUÇÃO}

A dor está presente na maioria dos processos fisiológicos que envolvem a relação saúde-doença. É uma experiência pessoal e complexa, e mensurá-la não é possível através de instrumentos físicos comumente utilizados na verificação dos demais sinais vitais. Apesar disso, reconhecer o fenômeno doloroso no âmbito hospitalar tornou-se indispensável para planejamento, tomada de decisões e avaliação da terapêutica proposta (SILVA MS et al., 2011).

Culturalmente a dor é expressa de maneira distinta e seu relato pode ser variado mesmo que os sujeitos vivenciem, teoricamente, a mesma sensação. Também é descrita e conhecida como fenômeno emocional, passível de influência de fatores externos e experiências anteriores, atuando em sua intensidade, manifestação e expressão (MELO LR e PETTENGILL MAM, 2010).

Por ter caráter subjetivo, ao avaliarmos a dor em recém-nascidos, crianças e adolescentes enfrentamos obstáculos relacionados à dificuldade de expressão, comunicação verbal e desenvolvimento do pensamento associativo infantil (SILVA FC e THULER LCS, 2008). Até metade de década de 1970 as crianças eram julgadas incapazes de quantificar fenômenos abstratos como a intensidade da dor (MELO LR e PETTENGILL MAM, 2010).

Diante desta realidade, torna-se cada vez mais evidente a necessidade de avaliar a dor por meio de um instrumento efetivo, considerando a faixa etária e manifestações específicas para cada idade baseadas em critérios como autor relato, observação e estado fisiológico (SILVA FC e THULER LCS, 2008).

No cenário deste estudo, a implantação do registro e da avaliação da dor como $5^{\circ}$ sinal vital a ser mensurado com a utilização de escalas/instrumentos padronizados ocorreu no ano de 2007 (NASCIMENTO LA e KRELING MCGD, 2011).

Posteriormente, em 2011, frente às dificuldades de mensurar a dor com os instrumentos disponíveis, voltados para a população adulta, desenvolveu-se um estudo quantitativo na unidade de terapia intensiva pediátrica (UTIP) deste hospital, envolvendo os membros da equipe de enfermagem, para implantação de escala adequada de avaliação da dor pediátrica. Os participantes, depois de instruídos quanto à utilização das escalas, responderam a um questionário sobre sua aplicabilidade, prática, clareza, compreensão, tempo de aplicação e dificuldades para utilização da mesma na rotina de trabalho diária (SILVA LDG et al., 2014).

Mediante avaliação dos resultados, a escala escolhida para implantação na unidade foi a Face, Legs, Activity, Cry, Consolability (FLACC), de caráter comportamental, avalia padrões de face, pernas, atividade, choro e consolabilidade. De fácil aplicabilidade mostrou-se efetiva quando utilizada para demonstrar a 
mudança no escore de dor antes e depois da administração de medicamentos analgésicos (SILVA FC e THULER LCS, 2008).

Este instrumento pode ser aplicado em crianças de dois meses a sete anos de idade e possibilita avaliação em condições de consciência ou inconsciência. A pontuação varia entre zero e 10 e a dor pode ser classificada conforme 0 escore em $0=$ Relaxado e confortável, $1-3=$ Desconforto médio, $4-6=$ Dor moderada, $7-10=$ Dor/desconforto severo (SILVA FC e THULER LCS, 2008).

A sensação dolorosa é temida pelas crianças edurante o período de internação, vivenciam procedimentos invasivos, agressivos e dolorosos, resultando em sensações como medo, ansiedade e resistência. Quando subtratada ou relegada a um segundo plano, deixa sequelas que poderão refletir em sua vida posterior (SILVA FC e THULER LCS, 2008).

A dor faz parte do processo de hospitalização infantil. Embora não possa ser totalmente evitada há meios para minimizá-la e tratá-la quando reconhecida de maneira precoce e efetiva. Reconhecer a dor em crianças é difícil, principalmente em situações de alta complexidade, neste contexto, a presente pesquisa teve por finalidade avaliar a utilização da Escala FLACC pela equipe de enfermagem como instrumento para manejo da dor de crianças internadas em uma UTIP.

\section{MÉTODO}

Estudo retrospectivo e descritivo, de abordagem quantitativa, realizado em um hospital universitário público, de nível terciário, localizado no sul do Brasil.

Os dados do estudo foram obtidos por meio da análise dos prontuários disponibilizados pela Divisão de Arquivo Médico e Estatística do hospital das crianças com idade entre zero dia de vida a sete anos incompletos, internadas na UTIP em dois períodos distintos: o primeiro, nos três meses imediatos à implantação da escala FLACC na unidade (novembro, dezembro de 2011 e janeiro de 2012) e o segundo, um ano após a implantação (novembro e dezembro de 2012 e janeiro de 2013). A coleta ocorreu no ano de 2016.

Após coleta realizou-se interpretação dos dados sociodemográficos e dos relativos à internação: diagnóstico médico, período de internação, uso de analgesia, identificação e classificação da dor por meio da escala implantada, registro em prescrição médica e relatórios de enfermagem, além da escolha terapêutica adotada e desfecho da internação. Os resultados foram analisados por intermédio do software IBM SPSS Statistics 20.

A pesquisa foi aprovada pelo Comitê de Ética em Pesquisa Envolvendo Seres Humanos da Universidade Estadual de Londrina - UEL, com parecer o 1.627.996, de 07 de julho de 2016, CAAE no 56739316.1.0000.5231.

\section{RESULTADOS}

Entre os períodos pesquisados foram hospitalizadas na UTIP 62 crianças, sendo 35 (54,45\%) do gênero masculino, com média de idade em 0,9 anos e 27 (45,55\%) crianças do gênero feminino, sendo a média de idade em 1,4 anos. Quanto à procedência, 28 crianças (45,16\%) atendidas eram residentes em Londrina e 34 (54,84\%) de outros municípios do Paraná.

Os atendimentos clínicos foram a principal causa de consultas seguidas de internação na referida unidade $(82,25 \%)$. As doenças do sistema respiratório destacaram-se nos diagnósticos médicos referidos para as crianças $(35,48 \%)$ (Tabela 1$)$.

O total de dias de internação variou entre um e 44, com média de 7,05 dias. Quanto ao desfecho, três $(4,83 \%)$ crianças tiveram alta com estado "curado", 46 (74,19\%) "melhorado", três (4,83\%) transferidos e 10 $(16,12 \%)$ evoluíram para óbito. 
Tabela 1 - Distribuição dos diagnósticos médicos das crianças entre zero e sete anos hospitalizadas na UTIP, nos períodos de 01 de novembro de 2011 a 31 de janeiro de 2012 e 01 de novembro de 2012 a 31 de janeiro de 2013 agrupados por capítulos do Código Internacional de Doenças (CID)10, no ano de 2016. Londrina, 2018.

\begin{tabular}{lll}
\hline Diagnósticos Médicos & N & \% \\
\hline Doenças do sistema respiratório & 22 & 35,48 \\
Malformações congênitas, deformidades e anomalias cromossômicas & 8 & 12,90 \\
Infecções e choque & 7 & 11,29 \\
Doenças do sistema renal e hepático & 4 & 6,45 \\
Doenças endócrinas, nutricionais e metabólicas & 1 & 1,61 \\
Traumatismo de região não especificada do corpo & 3 & 4,84 \\
Queimaduras e corrosões & 3 & 4,84 \\
Neoplasias & 3 & 4,84 \\
Intoxicação por drogas, medicamentos e substâncias biológicas & 2 & 3,23 \\
Doenças do aparelho circulatório & 2 & 3,23 \\
Doenças do sistema nervoso & 7 & 11,29 \\
\hline TOTAL & 7 & $\mathbf{1 0 0} \%$ \\
\hline
\end{tabular}

Fonte: produção do próprio autor.

As anotações de enfermagem foram avaliadas pela pesquisadora nos períodos matutino, vespertino e noturno, com o objetivo de identificar relato do quadro doloroso apresentado pela criança por meio de sinais e sintomas, nos casos em que a escala não tivesse sido utilizada. Imediatamente à implantação, em $31,82 \%$ dos prontuários foi possível encontrar relato do processo doloroso. Um ano após, apenas em 8,57\% foram encontrados na ausência da graduação da dor (Tabela 2 e 3).

Tabela 2 - Distribuição da frequência de crianças medicadas e não-medicadas no período entre 01 de novembro de 2011 e 31 de janeiro de 2012 por justificativa, no ano de 2016. Londrina, 2018.

\begin{tabular}{lcc}
\hline \multicolumn{1}{c}{ Variável } & N & $\%$ \\
\hline Crianças medicadas & 22 & 88 \\
Crianças não-medicadas & 3 & 12 \\
Total & $\mathbf{2 5}$ & $\mathbf{1 0 0}$ \\
\hline & & \\
Medicadas classificadas com pontuação na Escala FLACC & 10 & 45,45 \\
Medicadas sem pontuação, com justificava em anotação de enfermagem & 7 & 31,82 \\
Medicadas sem pontuação e sem justificativa & 5 & 22,73 \\
Total & $\mathbf{2 2}$ & $\mathbf{1 0 0}$ \\
\hline
\end{tabular}

Fonte: produção do próprio autor. 
Tabela 3 - Distribuição da frequência de crianças medicadas e não-medicadas no período entre 01 de novembro de 2012 e 31 de janeiro de 2013 por justificativa, no ano de 2016. Londrina, 2018.

\begin{tabular}{lcc}
\hline & $\mathbf{N}$ & $\%$ \\
\hline Crianças medicadas & 35 & 94,59 \\
Crianças não-medicadas & 2 & 5,41 \\
Total & $\mathbf{3 7}$ & $\mathbf{1 0 0}$ \\
\hline Medicadas classificadas com pontuação da Escala FLACC & 19 & \\
Medicadas sem pontuação, com justificava em anotação de enfermagem & 3 & 84,29 \\
Medicadas sem pontuação e sem justificativa & 13 & 37,14 \\
Total & $\mathbf{3 5}$ & $\mathbf{1 0 0}$ \\
\hline
\end{tabular}

Fonte: produção do próprio autor.

Quanto à terminologia, analisando simultaneamente os dois períodos, identificou-se em 31 (50\%) prontuários o termo "choro"; "agitado" em 24 (38,70\%); "febril" em dez (17,74\%); "fácies de dor" em cinco (8,06\%), "gemência" em seis $(9,68 \%)$ e "dor" em dois (3,23\%) relatos (Figura 1).

Figura 1 - Representação da terminologia.

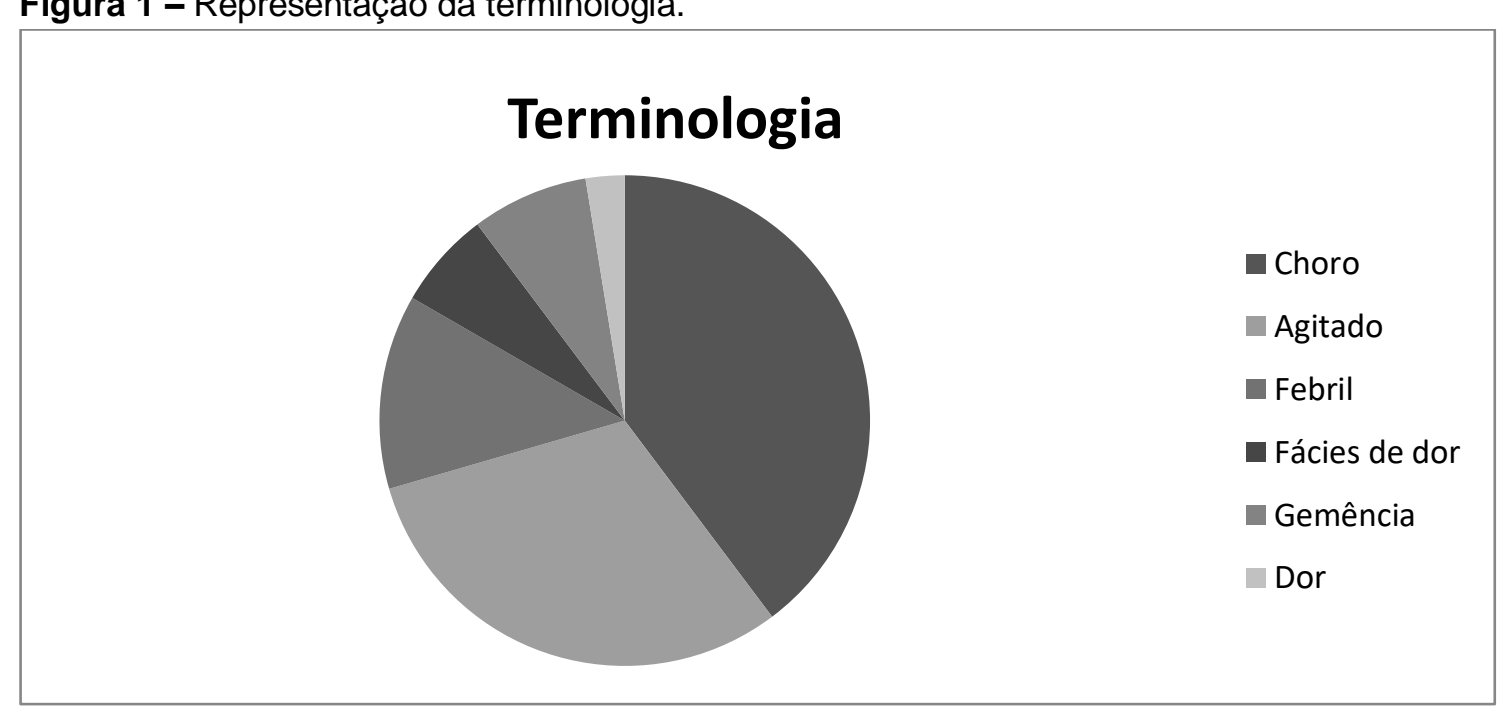

Fonte: produção do próprio autor.

Para o tratamento da dor, foram encontrados registros, em ambos os períodos, do uso de analgésicos de diferentes classes, para dores de baixa, moderada e alta intensidade, sem a descrição do critério da analgesia de escolha ou do seguimento da recomendação que sugere a Organização Mundial da Saúde (OMS). Na amostra, evidenciou-se que, em pelo menos um momento da internação, 42 (67,74\%) crianças receberam como medida farmacológica Fentanil, 40 (64,51\%) Dipirona, 22 (35,48\%) Cetamina, seis (9,68\%) Morfina, três $(4,84 \%)$ Tramadol e 16 (25,80\%) Paracetamol. Em nenhum prontuário analisado foi encontrado relato do uso de medidas não-farmacológicas para conforto e alívio da dor. 


\section{DISCUSSÃO}

Dentre os diagnósticos, as doenças do sistema respiratório foram responsáveis por mais de um terço dos leitos ocupados nos períodos analisados. Poucos estudos descrevem o perfil epidemiológico das internações em UTIP. Lanetzki CS, et al. (2012) relataram o perfil epidemiológico das internações na UTIP de um hospital da capital paulista no ano de 2009 e, assim como o identificado, a maior parte dos pacientes teve como motivos de internação diagnósticos clínicos $(75,75 \%)$ e as causas respiratórias ocuparam as três primeiras posições entre os principais diagnósticos (LANETZKI CS et al., 2012) (Tabela 1).

A identificação e o manejo adequado da dor são um desafio para os profissionais da saúde. Sabe-se que menos da metade dos pacientes tem controle e manejo adequados. As barreiras são diversas: conduta tomada, uso de protocolos sem evidência científica, resistência da equipe multiprofissional para mudar conduta, método inadequado de avaliação da dor e treinamento insuficiente dos profissionais (SAKATA RK, 2010).

Em relação aos períodos analisados, imediato e tardio à escala, nota-se que 0 índice de crianças medicadas classificadas e com pontuação na escala de dor manteve-se alto: 45,45\% imediatamente e 54,29\% um ano após. Entretanto, nota-se um aumento significativo nos registros de crianças que foram medicadas sem justificativas tanto com base na escala FLAAC, quanto nos relatórios de enfermagem, chegando a $37,14 \%$ da amostra do segundo período (Tabela 2 e 3 ).

A equipe de enfermagem encontra-se envolvida diretamente no cuidado ao paciente possibilitando ao profissional conhecê-lo em sua individualidade. Esta relação possibilita a identificação do surgimento de novos episódios dolorosos na criança, resultando em assistência efetiva, melhor tratamento e alívio (BLASI DGE et al., 2015).

A anotação de enfermagem, associada à evolução de enfermagem, é um meio de comunicação entre as equipes e permite, quando realizada de forma adequada, a compreensão dos processos relacionados ao paciente de forma cronológica e o desfecho de toda situação, além dos cuidados que foram prestados possibilitando avaliação das medidas tomadas e do processo de trabalho envolvido (SILVA JA et al., 2012).

Os resultados evidenciaram falha na anotação de enfermagem em relação aos eventos dolorosos ao identificar a ausência de relato de dor e escore no momento da realização dos sinais vitais em $22,73 \%$ da amostra, logo após a implantação da escala e em $37,14 \%$ no período seguinte.

Pesquisa realizada em unidade neonatal de um hospital no município de São Paulo - SP aponta que os métodos não-farmacológicos aplicados em pacientes internados que enfrentam o processo doloroso apresentam-se como alternativa eficaz no tratamento da dor (CRESCÊNCIO EP et al., 2009). Na prática diária é realizado, principalmente pela enfermagem, abrangendo medidas simples e de baixo custo: mudança de decúbito, massagem local e banho de imersão. Alternativas também podem ser utilizadas em crianças: sucção em seio materno, sucção não-nutritiva ou uso de glicose oral.

Estudo realizado anteriormente com a equipe de enfermagem na mesma UTIP, cenário desta pesquisa, constatou que a maioria dos profissionais referiu não utilizar métodos não-farmacológicos para controle da dor, fazendo uso apenas de medicação prescrita. Já os que afirmaram utilizar tais métodos, citaram exemplos como compressas, glicose a $25 \%$ por via oral, apoio emocional e posicionamento. Ademais, a maioria dos entrevistados referiu a não continuidade da mensuração deste sinal vital, não consideram, portanto, tal avaliação como importante para o bem-estar da criança (BLASI DGE et al., 2015).

Em revisão bibliográfica acerca dos cuidados de enfermagem e o manejo da dor em crianças hospitalizadas, concluiu-se que os profissionais atuantes eram capazes de reconhecer a dor na criança apesar de não utilizar métodos validados para mensurá-la. Mesmo comprometidos em identificar o processo doloroso, também existiam fragilidades quanto ao conhecimento relacionado ao seu controle, observadas no emprego apenas de métodos farmacológicos (SANTOS JP e MARANHÃO DG, 2016).

Em relação ao emprego de analgésicos para alívio da dor persistente nas crianças hospitalizadas, não há registros que demonstrem seu uso baseado nos conceitos-chave propostos pela World Health Organization 
(WHO) Guidelines on the Pharmacological Treatment of Persisting Pain in Children with Medical IIInesses (2012),acerca da estratégia analgésica baseada em dois passos no tratamento da dor leve, moderada e grave, de forma individualizada, considerando a dosagem em intervalos regulares e via de administração apropriada.

De acordo com as recomendações, paracetamol e ibuprofeno são os medicamentos de escolha no primeiro passo (dor leve). Se moderada ou grave, a administração de um opióide forte é necessário como morfina, medicamento de escolha para o segundo passo (WHO, 2012).

\section{CONCLUSÃO}

Embora existam instrumentos validados e implantados nas unidades assistenciais, nota-se que diversas barreiras são encontradas para que este processo seja realizado de modo satisfatório, evidenciados no cenário do estudo pelo baixo número de pontuações encontradas nos prontuários avaliados mesmo no período imediato a implantação da escala. A maioria dos profissionais que atua no cenário deste estudo não recebeu treinamento sobre a escala implantada na unidade, que vivencia uma alta rotatividade de equipe. Os resultados demonstraram a ausência de informações nos relatórios de enfermagem em geral, nos quais dados como a justificativa do uso de analgésicos foram ocultos, ademais não houve relatos de medidas nãofarmacológicas como alternativas para conforto e tratamento da dor. Por fim, evidencia-se a necessidade de orientação e capacitação da equipe de modo contínuo e periódico a fim de que os profissionais estejam aptos para reconhecer o processo doloroso e conduzi-lo de modo eficaz, oferecendo uma assistência qualificada e humanizada à criança criticamente enferma.

\section{REFERÊNCIAS}

1. BLASI DGE et al. Avaliação e manejo da dor na criança: percepção da equipe de enfermagem. Semina: Ciências Biológicas e da Saúde, 2015; 36(1): 301-310.

2. CRESCÊNCIO EP et al. Avaliação e alívio da dor no recém-nascido. Rev. Eletr. Enf., 2009; 11(1): 64-9.

3. LANETZKI CS et al. O perfil epidemiológico do centro de terapia intensiva pediátrico do hospital israelita Albert Einstein. Einsten, 2012; 10(1): 16-21.

4. MELO LR, PETTENGILL, MAM. Dor na infância: atualização quanto à avaliação e tratamento. Rev. Soc. Bras. Enferm. Ped. São Paulo, 2010; 10(2): 97-102.

5. NASCIMENTO LA, KRELING MCGD. Avaliação da dor como quinto sinal vital: opinião de profissionais de enfermagem. Acta Paul Enferm, 2011; 24(1): 50-54.

6. SAKATA RK. Analgesia e sedação em unidade de terapia intensiva. Rev. Bras. Anestesiol, 2010; 60(6): 653-658.

7. SANTOS JP, MARANHÃO DG. Cuidado de enfermagem e manejo da dor em crianças hospitalizadas: pesquisa bibliográfica. Rev. Soc. Bras. Enferm. Ped, 2016; 16(1): 44-50.

8. SILVA FC, THULER LCS. Tradução e adaptação transcultural de duas escalas para avaliação da dor em crianças e adolescentes. J. Pediatr., 2008; 84(4): 344-349.

9. SILVA JA et al. Avaliação da qualidade das anotações de enfermagem em unidade semi-intensiva. Esc. Anna Nery, 2012; 16(3): 576-581.

10. SILVA LDG, et al. Escalas de avaliação de dor: processo de implantação em uma unidade de terapia intensiva pediátrica. Rev enferm UFPE, 2014; 8(4): 857-63.

11. SILVA MS, et al. Dor na criança internada: a percepção da equipe de enfermagem. Rev. dor, 2011; 12(4): 314-320.

12. WORLD HEALTH ORGANIZATION (WHO) Guidelines on the Pharmacological Treatment of Persisting Pain in Children with Medical Illnesses. Geneva: World Health Organization, 2012. 